Louisiana State University

LSU Digital Commons

Faculty Publications

Department of Biological Sciences

3-28-2016

\title{
Discovery of Novel Nonactive Site Inhibitors of the Prothrombinase Enzyme Complex
}

\author{
Karan Kapoor \\ The University of Tennessee, Knoxville \\ Nicole McGill \\ Shifa Biomedical Corporation \\ Cynthia B. Peterson \\ The University of Tennessee, Knoxville \\ Harold V. Meyers \\ Shifa Biomedical Corporation \\ Michael N. Blackburn \\ Shifa Biomedical Corporation
}

See next page for additional authors

Follow this and additional works at: https://digitalcommons.Isu.edu/biosci_pubs

\section{Recommended Citation}

Kapoor, K., McGill, N., Peterson, C., Meyers, H., Blackburn, M., \& Baudry, J. (2016). Discovery of Novel Nonactive Site Inhibitors of the Prothrombinase Enzyme Complex. Journal of Chemical Information and Modeling, 56 (3), 535-547. https://doi.org/10.1021/acs.jcim.5b00596

This Article is brought to you for free and open access by the Department of Biological Sciences at LSU Digital Commons. It has been accepted for inclusion in Faculty Publications by an authorized administrator of LSU Digital Commons. For more information, please contact ir@lsu.edu. 


\section{Authors}

Karan Kapoor, Nicole McGill, Cynthia B. Peterson, Harold V. Meyers, Michael N. Blackburn, and Jerome Baudry 


\title{
Discovery of Novel Nonactive Site Inhibitors of the Prothrombinase Enzyme Complex
}

\author{
Karan Kapoor, ${ }^{\dagger, \S, \nabla}$ Nicole McGill, ${ }^{\|, \nabla}$ Cynthia B. Peterson, ${ }^{\ddagger}$ Harold V. Meyers, ${ }^{\|}$Michael N. Blackburn, ${ }^{*}, \|$ \\ and Jerome Baudry*,\$, \\ ${ }^{\dagger}$ UT/ORNL Program in Genome Science and Technology, Knoxville, Tennessee 37830, United States \\ ${ }^{\ddagger}$ Department of Biochemistry and Cellular and Molecular Biology, University of Tennessee, Knoxville, Tennessee 37996, United \\ States \\ ${ }^{\S}$ UT/ORNL Center for Molecular Biophysics, Oak Ridge, Tennessee 37830, United States \\ "Shifa Biomedical, One Great Valley Parkway, Suite 8, Malvern, Pennsylvania 19355, United States
}

Supporting Information

\begin{abstract}
The risk of serious bleeding is a major liability of anticoagulant drugs that are active-site competitive inhibitors targeting the Factor $\mathrm{Xa}(\mathrm{FXa})$ prothrombin (PT) binding site. The present work identifies several new classes of small molecule anticoagulants that can act as nonactive site inhibitors of the prothrombinase (PTase) complex composed of FXa and Factor $\mathrm{Va}$ (FVa). These new classes of anticoagulants were identified, using a novel agnostic computational approach to identify previously unrecognized binding pockets at the FXa-FVa interface. From about three million docking calculations of 281128 compounds in a conformational ensemble of FXa heavy chains identified by molecular dynamics (MD) simulations, 97 compounds and their structural analogues were selected for experimental validation, through a series of inhibition assays. The compound selection was based on their predicted binding affinities to FXa and their ability to successfully bind to multiple protein conformations while showing selectivity for particular binding sites at the FXa/FVa interface. From these, thirty-one (31) compounds were experimentally identified as nonactive site inhibitors. Concentrationbased assays further identified 10 compounds represented by four small-molecule families of inhibitors that achieve doseindependent partial inhibition of PTase activity in a nonactive site-dependent and self-limiting mechanism. Several compounds were identified for their ability to bind to protein conformations only seen during MD, highlighting the importance of accounting for protein flexibility in structure-based drug discovery approaches.
\end{abstract}

\section{INTRODUCTION}

The blood coagulation system represents the first line of defense against blood loss following injury. This system consists of a cascade of circulating inactive serine protease zymogens, as well as regulatory cofactors and inhibitors. Each active enzyme, once generated from its zymogen, specifically cleaves the next zymogen in the cascade pathway to produce an active protease, a process that is repeated until, finally, thrombin cleaves the fibrinopeptides from fibrinogen to produce fibrin, which polymerizes to make a blood clot. The amplification provided by this system leads to the potential for explosive clot formation. ${ }^{1}$ Although efficient clotting limits the loss of blood at a site of trauma, it also poses the risk of systemic coagulation, resulting in massive thrombosis. This can result in myocardial infarction, unstable angina, atrial fibrillation, stroke, pulmonary embolism and deep vein thrombosis. Several new direct Factor $\mathrm{Xa}$ (FXa) inhibitors have received approval for clinical use in anticoagulant therapy. Each of these drugs binds in the active site of the coagulation protease and is a competitive inhibitor of FXa.

The prothrombinase (PTase) enzymatic complex, consisting of the enzyme FXa, a protein cofactor, Factor Va (FVa), associated on the surface of negatively charged phospholipid membranes in the presence of divalent metal ions, ${ }^{2,3}$ forms a critical junction of the blood coagulation cascade pathways. ${ }^{4}$ This complex catalyzes the cleavage of prothrombin (PT), leading to the formation of thrombin and subsequent clot formation. ${ }^{5}$ FXa alone can slowly activate PT, but the rate of thrombin formation is enhanced by a factor of $>10^{5}$ by the presence of the cofactor, $\mathrm{FVa}$, and $\mathrm{Ca}^{2+}$ on phospholipid membranes in the PTase complex. ${ }^{6,7}$

FXa consists of two chains: the light chain of 139 residues, and the heavy chain of 305 residues, which contains the catalytic active site and is connected via a disulfide $\left(\mathrm{Cys} 122_{\mathrm{H}^{-}}\right.$

Received: September 28, 2015

Published: February 5, 2016 

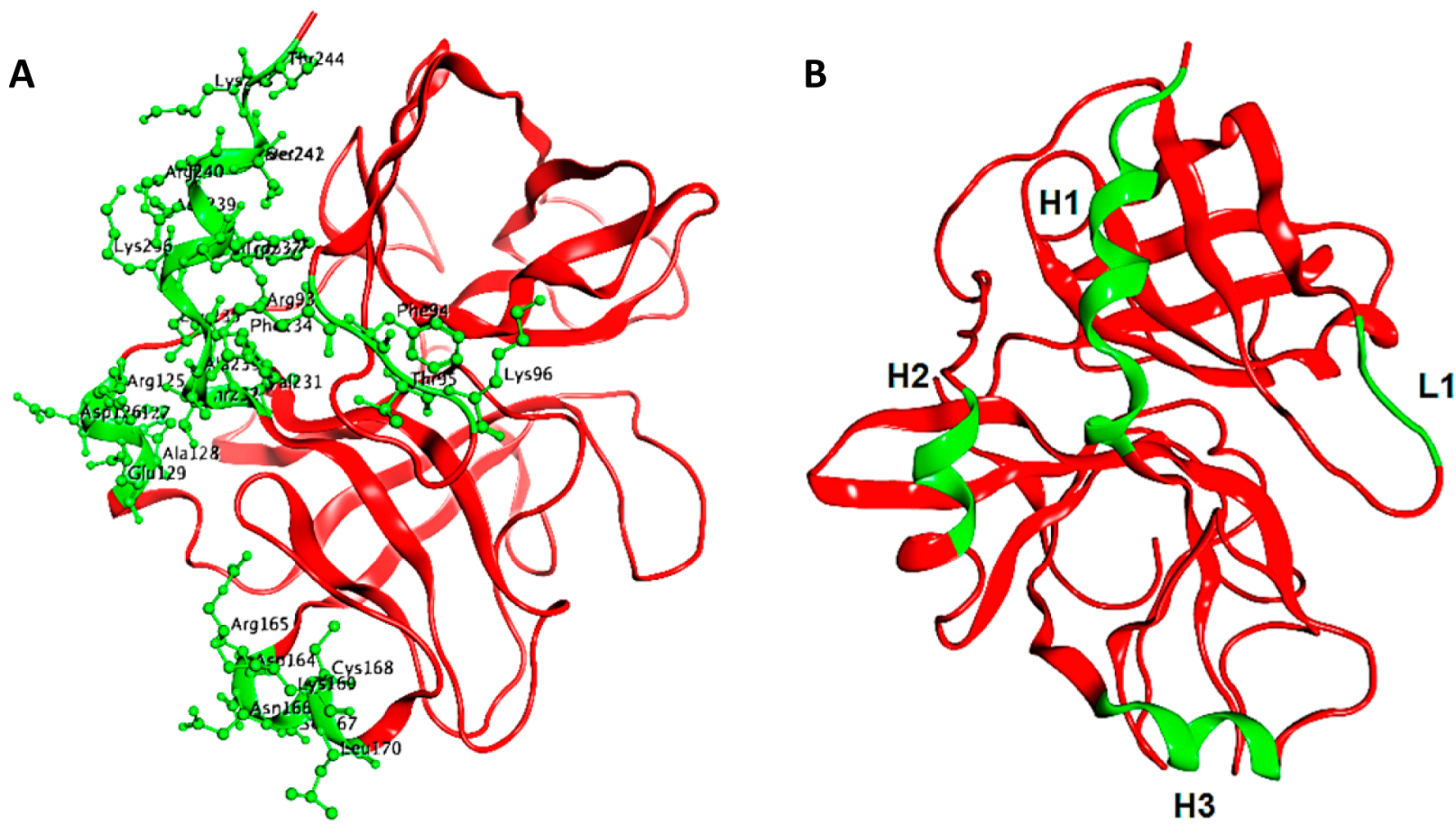

H3

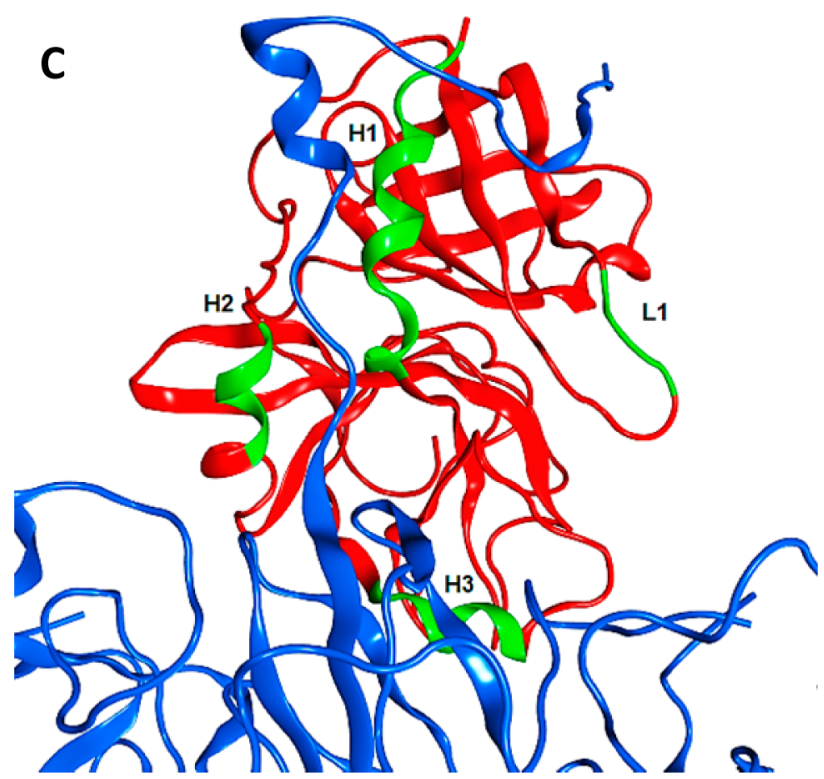

Figure 1. FXa-FVa binding site: (A) human FXa residues proposed to interact with human FVa, based on the FVa-FXa complex models and the available experimental data (binding site residues 93-96, 125-129, 164-170, 231-244 are shown in green); (B) residues (green) form part of 3 helices ( $\mathrm{H} 1, \mathrm{H} 2$, and $\mathrm{H} 3$ ) and a loop (L1); (C) superposition of human FXa with the FX-FV complex from P. textilis (only superposed human FXa (red) and $P$. textilis FV (blue) are shown, and the P. textilis FX structure is not shown for the sake of clarity; the binding interface (shown in green) in human FXa is proposed to interact with human FVa).

Cys $132_{\mathrm{L}}$ ) bridge to the light chain. ${ }^{8}$ A wealth of structural data is available for this protein; over 150 crystal structures of the protein or protein-ligand complexes have been deposited in the Brookhaven Protein Data Base (PDB). ${ }^{9}$ Activated human FVa consists of a noncovalent calcium-dependent complex between the heavy chain (Hc: A1 (1-303)-A2 (317-656) domains) and the light chain (Lc: A3 (1546-1877)-C1 (1878-2036)-C2 (2037-2196) domains), in which the cofactor binding site is exposed for interaction with FXa. ${ }^{10,11}$ Several studies have shown that both the Hc and Lc of FVa are involved in the binding of FXa, and they also give some insights about FVa residues interacting with $\mathrm{FXa}^{12-16}$ In addition, some regions in the $\mathrm{Hc}$ of $\mathrm{FXa}$ have been suggested to be important for the interaction with FVa. ${ }^{17-23}$ High-resolution crystal structures of FXa are available, ${ }^{24}$ and homology models of FVa using the crystal structure of inactivated bovine FVa $(\text { FVai })^{25}$ have also been built. Using these two structures, models of the FXa-FVa complex have been generated that are consistent with available experimental data. ${ }^{26}$

The goal of this study is to identify FXa inhibitors that function within the PTase complex; however, instead of seeking another active-site directed inhibitor, high-throughput virtual 


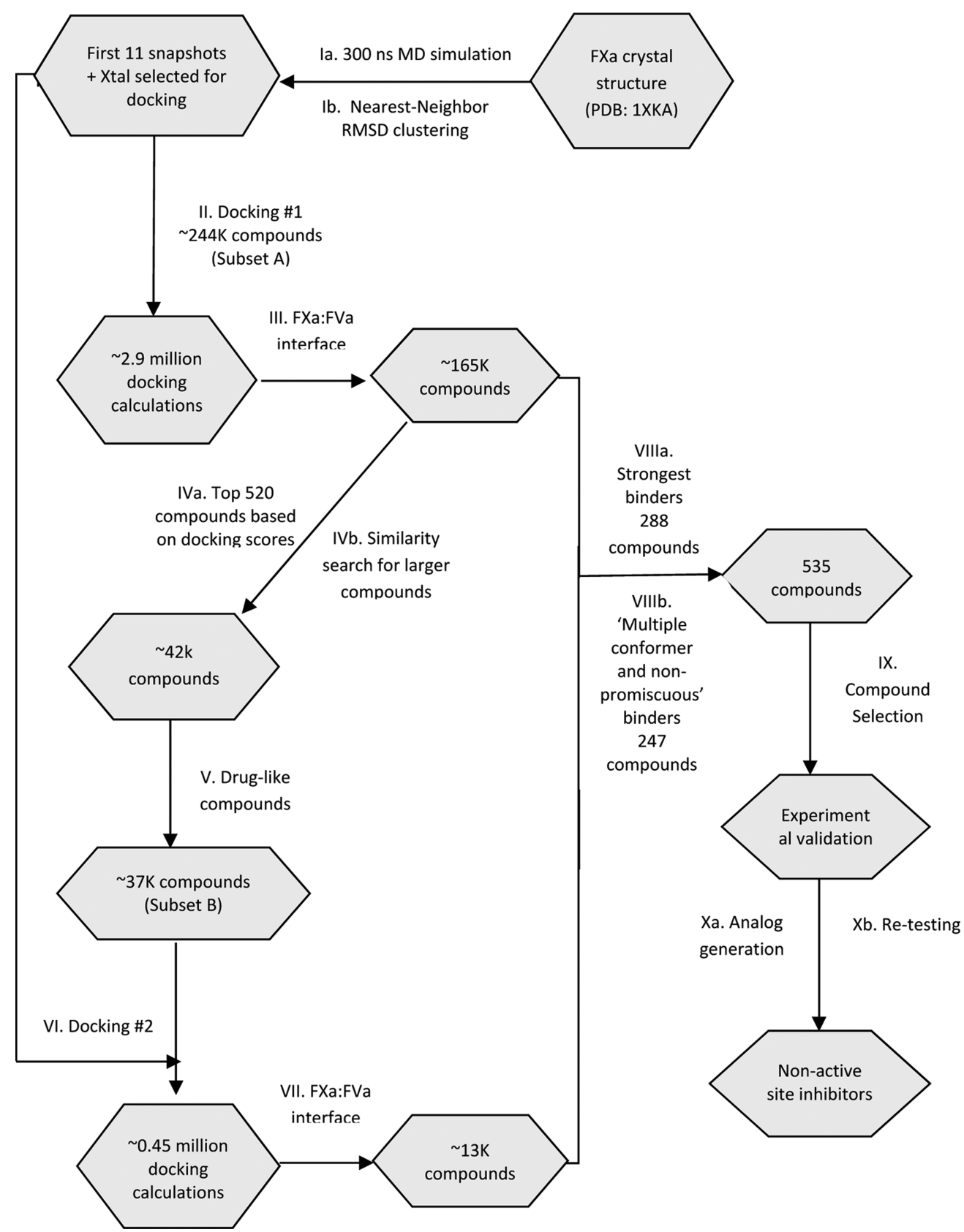

Figure 2. Flowchart showing the Ensemble docking process. Step I: $300 \mathrm{~ns}$ MD simulation was performed (pathway a), followed by nearestneighbor RMSD clustering (pathway b), generating 15 snapshots. Step II: 244000 compounds docked, covering $90 \%$ of 11.1 million "clean druglike" subset from the ZINC database. Step III: Parsed for compounds that bind to the FXa/FVa interface in the crystal structure or at least one of the 11 snapshots; only 532 out of 244493 docked compounds bind at this interface in the crystal structure. Step IV: Top 80 compounds in the crystal structure, plus top 40 compounds in each of 11 snapshots selected ( 520 total) (pathway a) for pharmacophore search with ZincPharmer for larger compounds (molecular weight (MW) of 280-600 amu), against purchasable ZINC database compounds (pathway b). Step V: Compounds parsed for druglike properties and redundant compounds removed. Step VI: 37000 larger compounds docked in 11 snapshots plus crystal structure. Step VII: Parsed again for compounds that bind to the FXa/FVa interface in the crystal structure or at least one of the 11 snapshots; only 177 out of 36635 docked larger compounds bind at this interface in the crystal structure. Step VIII: Top 50 compounds in crystal structure, plus top 30 compounds from each of 11 snapshots based on docking scores selected: 288 compounds (after removing redundancy), from which 44 bind to the crystal structure and others bind to MD snapshots (a); 247 compounds selected that bind to most number of snapshots and only 1 or 2 different binding sites on the FXa/FVa interface (pathway b). (Out of these, 79 bind to the crystal structure and the others bind to MD snapshots.) Step IX: Compound selection based on compounds' availability, cost, chemical diversity, and lack of reactive functional groups. Step X: Analog generation based on initial positive hits (pathway a); experimental validation of these analogues (pathway b).

screening methods are used to identify compounds that are likely to bind to FXa and alter the interaction between FXa and FVa, and hence have the potential to down-modulate the PTase activity in an nonactive site and noncompetitive manner.

\section{MATERIALS AND METHODS}

FXa-FVa Binding Interface. The active site-inhibited human FXa structure, solved by X-ray crystallography (PDB code: 1XKA), was used as the starting structure. ${ }^{24}$ It has a $2.3 \AA$ 
resolution and no gaps in the structure. MD simulations in previous studies showed that the light chain, which is connected to a negatively charged phospholipid membrane in the PTase complex and does not form any interactions with $\mathrm{FVa}$, is highly flexible in aqueous solution and undergoes angstroms-wide fluctuations within a few nanoseconds of the $\mathrm{MD}{ }^{27}$ To focus on the serine protease domain that interacts with FVa, this flexible part was removed and only the globular heavy chain was used in the MD calculations. Homology models of FVa using the crystal structure of inactivated bovine FVa (FVai) have been built by other groups. ${ }^{25}$ Using these models and the crystal structure of FXa, models of the FXaFVa complex have been generated that are in agreement with available experimental data. ${ }^{26}$ These models and the available experimental data were used to identify the regions of FXa that have been suggested to be important for the interaction with FVa.

Figure 1A shows the FXa binding surface that is proposed to interact with FVa, based on the FXa-FVa complex models, consisting of 28 residues. They form part of 3 helices $(\mathrm{H} 1, \mathrm{H} 2$, and H3) and one loop (L1), shown in Figure 1B.

Recently, the crystal structure of the FX-FV complex from the venom of Pseudonaja textilis has been solved. ${ }^{28}$ The complex shows specific interactions between the structured A2 and A3 domains of FV and FX. The highly flexible terminal region following the A2 domain forms nondirectional/nonspecific ionic interactions and lacks a fixed binding mode, and only a small hydrophobic portion at the end of this region (IFADIFI) was well-resolved.

The FXa binding interface selected in the current study can be compared to the crystal structure of the FX:FV complex from $P$. textilis. Human FX and FV sequences show an identity of $50.5 \%$ and $53.5 \%$ with the respective $P$. textilis sequences. The aligned structures of human FXa and $P$. textilis FX exhibit a root-mean-square deviation (RMSD) of $2.82 \AA$ (see the Supporting Information). Figure 1C shows the superposition of the human FXa crystal structure with the FX-FV complex from $P$. textilis (PDB code: 4BXS). The selected human FXaFVa binding site consisting of 3 helices and 1 loop also forms the interface between FX:FV complex from $P$. textilis (Figure 1C). The FXa-FVa contact region mapped on human FXa (and shown in Figure 1A) is essentially reproducing the $P$. textitlis protein/protein interface.

Molecular Dynamics (MD) Simulations and Clustering. The dynamics of the FXa heavy chain was characterized with MD simulations, using the NAMD2 simulation engine. ${ }^{29}$ The active site ligand and the light chain were removed from the model. One $\mathrm{Ca}^{2+}$ ion, which was known to be structurally important, ${ }^{30,31}$ was kept in the structure. The structure was protonated according to estimated $\mathrm{p} K_{\mathrm{a}}$ calculations, using the Protonate-3D facility in MOE, version 2011, ${ }^{32}$ with an ionic concentration of $0.1 \mathrm{~mol} / \mathrm{L}$ in the Generalized Born/Volume Integral (GB/VI) electrostatics model. ${ }^{33}$ A periodic solvation cube of water molecules was created using the Solvate facility in MOE-2011. Seven negative $\mathrm{Cl}^{-}$ions were added to neutralize the system, and the final system consisted of 22516 atoms, with 6279 water molecules. The system was energy-minimized to a gradient of $10^{-5} \mathrm{RMS} \mathrm{kcal} / \mathrm{mol} / \AA^{2}$, using the CHARMM22 force field $^{34}$ and an 8-10 $\AA$ nonbonded cutoff. NAMD2 input files were generated using the Dynamics facility in MOE-2011. A MD integration time step of 2 fs/step was used, holding covalent bonds in water molecules rigid. A constant temperature of $300 \mathrm{~K}$ was maintained throughout the simulation, using
Langevin dynamics. Particle Mesh Ewald (PME) was used for calculation of the electrostatic interactions with the periodic boundary conditions. Simulations were performed in the NVT ensemble. The MD simulation was run on the University of Tennessee High Performance Computing architecture (Newton). A 100 ps initial equilibration was performed, followed by a $300 \mathrm{~ns}$ production trajectory. The resulting trajectories were analyzed for convergence of temperature and RMSD using perl scripts for parsing data: Prody v1.2 ${ }^{35}$ and Gnuplot v4.6.

Structures of the protein along the MD trajectory were generated every 50 ps using Prody v1.2, i.e., generating a total of 6000 structures. Nearest-neighbor RMSD clustering was used to cluster these structures with Maxcluster, ${ }^{36}$ using the RMSD distance between the data point represented by each structure. Two structures were considered part of the same cluster if they were closer to each other than the cutoff threshold distance (i.e., if they were nearest-neighbors). The entire ensemble docking process flowchart is shown in Figure 2.

High-Throughput Docking Approach. Virtual screening was performed using the program Autodock Vina ${ }^{37}$ on 12 structures: 11 structures obtained from the MD clustering described above, and the FXa crystal structure. The "90\% similarity" "clean drug-like" subset of the ZINC database, ${ }^{38}$ with reactive functional groups removed, and that does not violate any of the Lipinski's criteria, was used for virtual screening. This subset contained 244493 compounds (referenced hereafter as "Subset A"), covering $90 \%$ of the $\sim 11$ million "clean drug-like" compounds of the ZINC database's chemical diversity, based on Tanimoto's similarity coefficient, ${ }^{39,40}$ and was precomputed and directly available from the ZINC database. The procedure used for clustering the molecules present in this subset involved sorting the ligands by increasing molecular weights and progressively selecting compounds that differ from those previously selected by at least the Tanimoto cutoff ( $90 \%$ in this case).

These ZINC compounds were converted from the smile format to the PDB format, using Babel v2.3.34 with the Generate-3D option. The $\mathrm{H}$ atoms in the molecules were further optimized in MOE-2011, rebalancing the protonation states by deprotonating strong acids and protonating strong bases by adding explicit $\mathrm{H}$ atoms, if needed. The geometries of the ligands were optimized using the MMFF94 force field ${ }^{42}$ in Babel. The compounds' coordinates in PDB format were converted to PDBQT format, using the Prepare Ligand scripts provided in ADT/MGL Tools v1.5.4. ${ }^{43}$ The FXa structures in $\mathrm{PDB}$ format from the clustered $\mathrm{MD}$ trajectory were also converted to PDBQT format directly in ADT tools. The configuration files for each FXa structure, including the docking grid box dimensions and the box center, used as input for docking in Autodock Vina, were created using ADT tools.

The Subset A compounds were docked in the crystal structure and in the first 11 representative structures from $\mathrm{MD}$ trajectory clustering, as indicated in Figure 2, allowing additional conformations of the ligand by sampling the rotatable bonds, and keeping the protein's side chains rigid. The docking was performed "agnostically," i.e., binding could happen anywhere on the protein without imposing a particular binding site using Autodock Vina on the Newton cluster. Default values were used for the maximum number of binding modes to be generated. The value of the exhaustiveness parameter in Autodock Vina, which directly determines the extent of sampling of chemical space in the binding site, was set to a value of 100 . Further details about the initial validation 
process of docking sampling/accuracy with docking speed are given in the Supporting Information.

Docking Results and Secondary Compound Selection. Perl scripts were used to select the best-scoring binding mode reported in the AutoDock Vina output for each ligand and to parse these binding modes in order to identify ligands that bind in the regions of FXa involved in FVa interactions (Step III in Figure 2).

The top 80 ranked compounds in the crystal structure and top 40 ranked compounds in each of the other $11 \mathrm{MD}$ snapshots (representing a total of 520 compounds) were identified from the docking calculations. ZincPharmer, ${ }^{44}$ which is a pharmacophore search tool for screening the ZINC database, was used to identify an additional 42309 compounds in the ZINC database that are chemically similar to the docking hits but with larger molecular weights (Step IV in Figure 2). These compounds were parsed to keep only the drug-like compounds with properties as defined in the ZINC database, i.e., $\mathrm{MW}=150-500, x \log P \leq 5$, net charge between -5 and 5 , $\leq 8$ rotatable bonds, polar surface area of $<150 \AA^{2}$, number of $\mathrm{H}$ donors $\leq 5$, number of $\mathrm{H}$ acceptors $\leq 10$, polar desolvation energy between $-400 \mathrm{kcal} / \mathrm{mol}$ and $1 \mathrm{kcal} / \mathrm{mol}$, apolar desolvation energy between $-100 \mathrm{kcal} / \mathrm{mol}$ and $40 \mathrm{kcal} / \mathrm{mol}$, and also, to remove any duplicates, giving a total of 36635 compounds (referenced hereafter as "Subset B"), corresponding to Step V in Figure 2. These compounds were again docked in the $12 \mathrm{MD}$ snapshot structures, using Autodock Vina on the UTK's Newton High Performance cluster with the same configuration settings as described above. Perl scripts were again used to parse the docking results for compounds that bind in the regions of FXa involved in FVa interactions.

Shortlisting Compounds for Experimental Testing. The results from docking of Subset A (Step II in Figure 2) and Subset B (Step VI in Figure 2) indicated that compounds can bind in different sub-binding sites at the $\mathrm{FXa} / \mathrm{FVa}$ interface, in different protein $\mathrm{MD}$ snapshots. The $\mathrm{FXa} / \mathrm{FVa}$ interface was divided into 10 sub-binding sites, based on the clustering of the binding loci for these compounds.

The computational hits were analyzed using two criteria (in addition to their predicted binding energies): (i) the number of MD snapshots to which one specific compound is predicted to bind, and (ii) the number of sites in the protein where a compound is predicted to bind. This allows the identification of compounds that bind to a large number of target structural variations and binding site(s), or, in other words, to quantify the promiscuous or specific character of a given compound, capable of binding to many protein conformations or to only a few. A value called the "snapshot count" was calculated that gives the total number of snapshots in which a given compound binds to the FXa/FVa interface. Compounds found to bind to most snapshots were checked for the different sub-binding sites (on the FXa/FVa interface), yielding another value, called the "binding-site score", which gives the number of sub-binding sites that a given compound is docked.

Two lists of compounds were generated for experimental validations: first, a list that contained 288 compounds, consisting of the strongest binders in the crystal structure, and the 11 snapshots, as predicted by Vina docking scores (shown in Step VIIIa in Figure 2). Second, a list was generated that contains 247 compounds with "snapshot count" scores of three and above (i.e., including compounds that successfully bind to three or more different protein conformations) and "binding-site scores" of one or two (i.e., removing promiscuous compounds that bind in many sites in different snapshots), shown in Step VIIIb of Figure 2. The first list thus contains compounds that are predicted to bind with the highest binding affinities at the FXa/FVa interface, and the second list contains the compounds that can bind to different protein conformations but, at the same time, are selective for particular binding sites at the interface.

Visual inspection was performed on the compounds in the two lists to assess their commercial availability and cost, and to identify and remove compounds with reactive functional groups (e.g., aldehydes and alkyl bromides), excessive hydrophobicity $(\log P>5)$, a lack of functionalization (e.g., polyaromatics with few or no functional groups) or an excess of functionalization, the latter two of which would render synthetic analoging difficult for any follow-up structure-activity relationship (SAR) studies. The compounds ordered for experimental testing were selected to sample different sub-binding sites at the interface, as predicted by the docking, having affordable cost and presenting desirable chemical features.

Experimental Validation. Structural analogues of the identified hits were searched from the list of all the compounds used for docking, including Subsets A and B, using Tanimoto's coefficient and the MACCS structural keys fingerprints ${ }^{45}$ in MOE-2011. Similar compounds not present in this list were also identified directly from the ZINC database, using substructure searching. Further filters described above were also applied on these compounds to order a second set of compounds. All compounds were dissolved in $10 \mathrm{mM}$, anhydrous, spectroscopic-grade dimethyl sulfoxide (DMSO) and were stored in sealed vials at $-20{ }^{\circ} \mathrm{C}$. Compounds were diluted in the assay to final concentrations of 5-100 $\mu \mathrm{M}$. The final DMSO concentration in the assay was kept constant at $1 \%$.

Initial screening of these compounds for potential FXa inhibitors was performed using a prothrombinase assay. ${ }^{6}$ FXa, FVa, prothrombin, and thrombin were purchased from Hematologic Technologies. The thrombin and FXa substrates (S-2238 and S-2222, respectively) were obtained from diaPharma. Apixaban was purchased from Selleckchem. PTase assays were performed in $50 \mathrm{mM}$ HEPES, $150 \mathrm{mM} \mathrm{NaCl}, 0.6$ $\mathrm{mM} \mathrm{MgCl}, 1.6 \mathrm{mM} \mathrm{CaCl}_{2}$, and 1\% PEG 6000 at $\mathrm{pH}$ 7.4. The final concentrations of FXa and FVa were $1 \mathrm{nM}$ and $10 \mathrm{nM}$, respectively, and the prothrombin substrate concentration was $1.4 \mu \mathrm{M}$. PTase activity was measured in a continuous assay that was initiated by the addition of the substrate prothrombin to the reaction mixture, and the rate of thrombin generation was determined by measuring hydrolysis of the thrombin chromogenic substrate, S-2238 $(1 \mathrm{mM})$ to produce $p$ nitroaniline (pNA) that was detected at $405 \mathrm{~nm}$. Replicate samples were analyzed and the rates were corrected for the very low rate of hydrolysis of S-2238 by FXa. For this two-step cascade reaction, in which the product of the first reaction is the enzyme of the second reaction, ${ }^{46}$ the activity of the first enzyme in this cascade (i.e., FXa.FVa) is proportional to $\mathrm{d} A_{405} / \mathrm{d} t^{2}$ and the absorbance vs time progress curves were fitted to a quadratic equation, using GraphPad Prism 5.0f. The activity of isolated FXa was measured using the substrate S-2222.

Binding Sites Analysis. The initial FXa inhibitors identified by PTase activity assays (and given in the Results section) were redocked in the binding sites, using MOE-2011 with the CHARMM27 Force-field, allowing protein side chain flexibility. Additional conformations of the ligand were allowed by sampling the rotatable bonds. Binding site residues side 


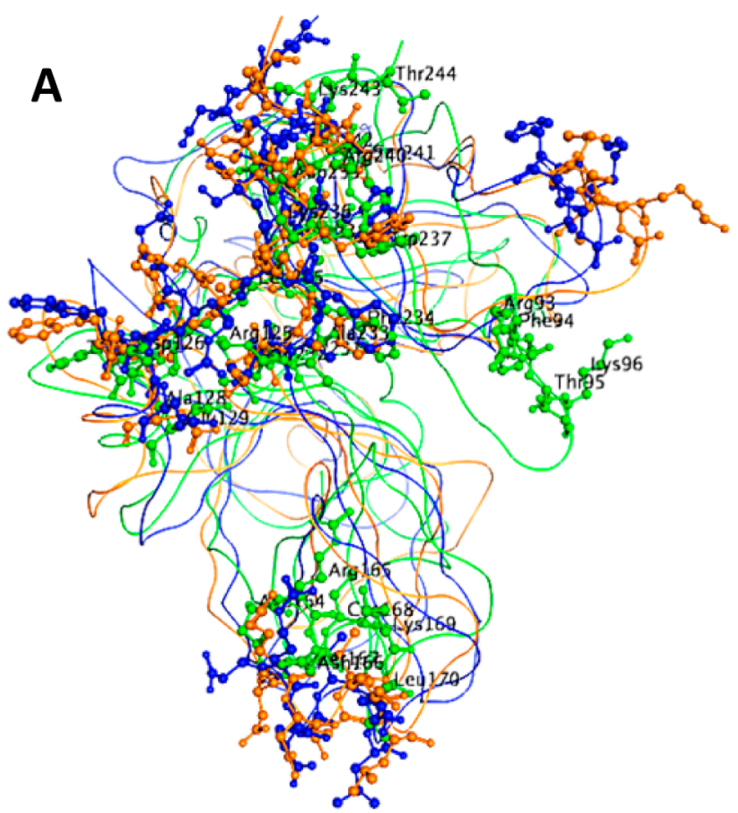

B
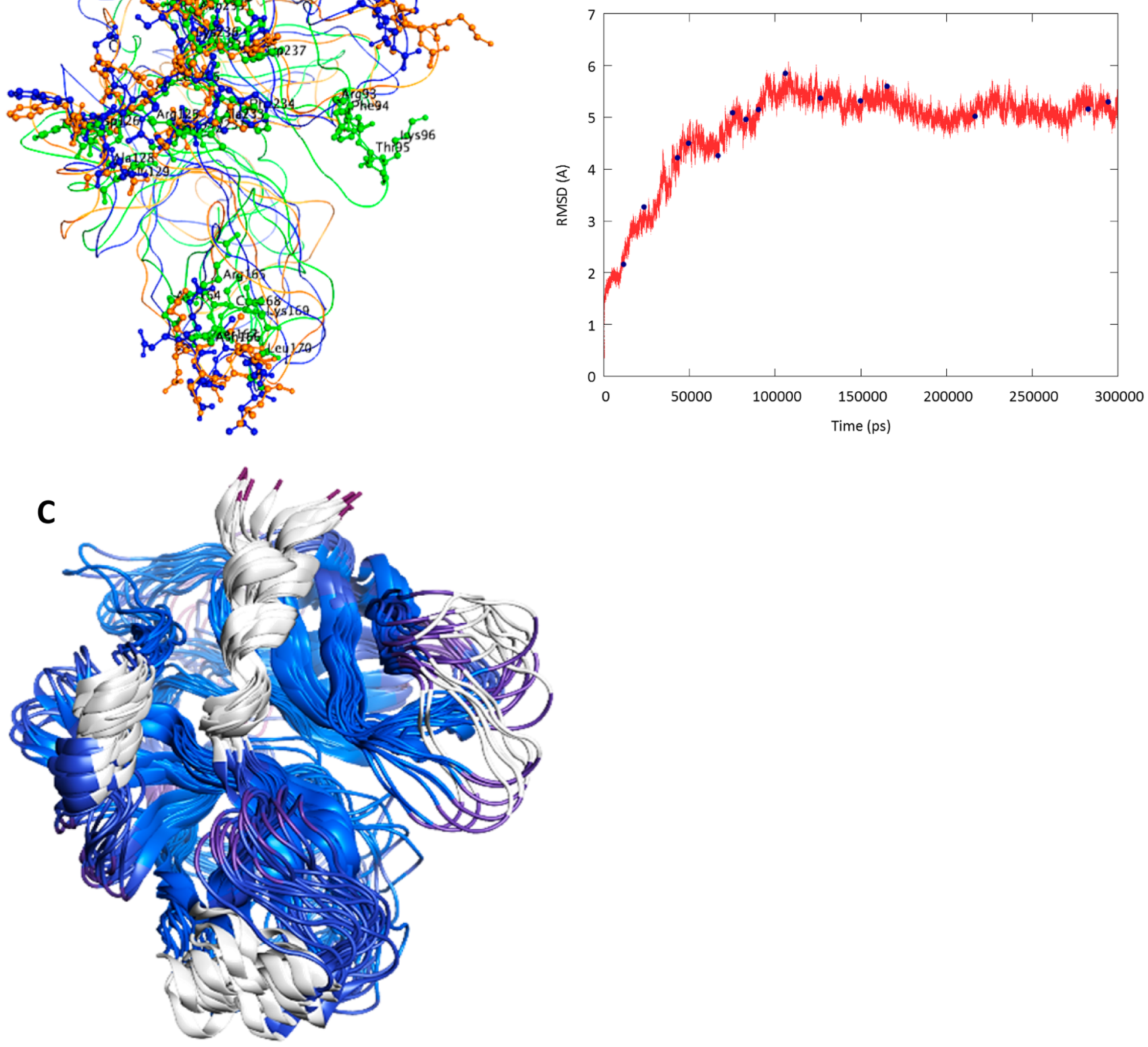

Figure 3. MD Simulations and Clustering. (A) Superposition of $3 \mathrm{FXa}$ structures at different times in the MD trajectory (starting crystal structure is shown in green, structure at $150 \mathrm{~ns}$ is shown in blue, and structure at $300 \mathrm{~ns}$ is shown in orange, with binding site residues shown as stick-ball representations). (B) RMSD plot over the time course of the trajectory. (C) Superposition of representative nonredundant structures from 15 clusters with binding site residues shown in white. Structures in panel (C) are represented on the RMSD plot in panel (B) as black dots.

chains were allowed to move away from their original location, using a tethering weight of 0.1 . The GBVI/WSA dG scoring function $^{47}$ was used for scoring different poses of the docked ligand. The top ranked binding poses were energy-minimized and the compounds' predicted binding free energies were calculated using a MM/GBVI-adapted protocol. ${ }^{33}$

\section{RESULTS}

MD Simulations and Clustering. The FXa heavy chain structure including the $\mathrm{FXa} / \mathrm{FVa}$ binding interface residues was found to sample different conformations in the MD snapshots, as shown in Figure 3A. Figure 3B shows the RMSD plot for the protein backbone over the time course of the trajectory.
Generally, MD simulations are considered stable when the backbone RMSD is in the low angstrom area. ${ }^{27}$ However, in the case of FXa, higher RMSD values (in the range of 2-7 $\AA$ ) have been reported. ${ }^{48-50}$ Based on the RMSD plot in Figure $3 \mathrm{~B}$, the FXa structure converges after the first $100 \mathrm{~ns}$ of the trajectory.

Nearest-neighbor RMSD clustering was used to divide the trajectory into 15 clusters as described in the Materials and Methods section. Figure 3C shows the superposition of these structures, also represented on the RMSD plot (Figure 3B) over the time course of the trajectory. Because of the constraints on the available computing time, only the first 11 of these clusters were used for docking calculations. 

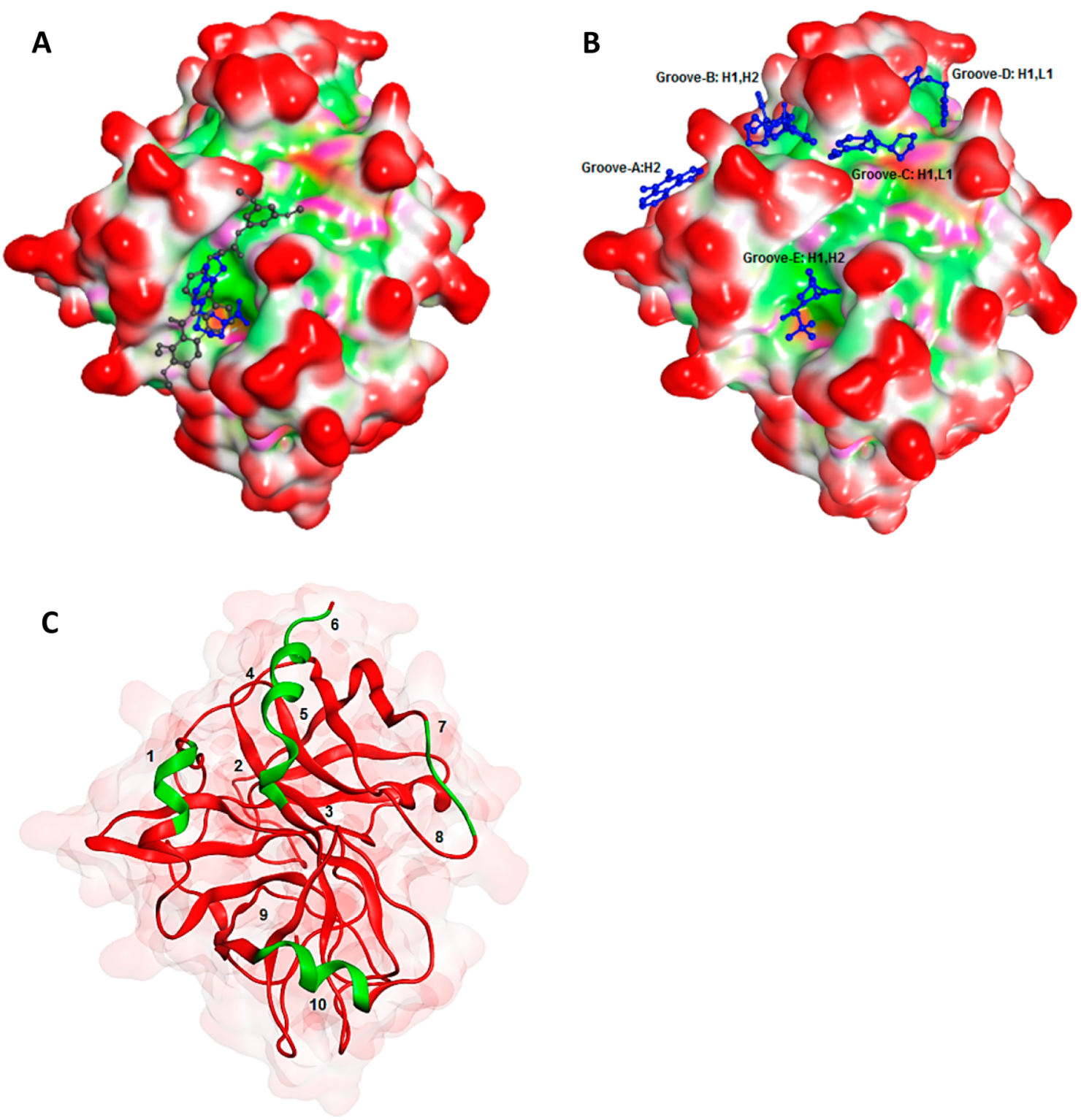

Figure 4. Docking analysis. (A) Predicted binding modes of smaller original compound from the Subset A in blue (SBC-160,014) and a larger similar compound from the Subset B in brown (SBC-160,045) binding in the FXa/Va interface in the crystal structure. The solvent exposed regions are shown as red on the molecular surface, hydrophobic regions are shown in green, and polar regions are shown in magenta. Larger compounds cover more binding surface. (B) In the crystal structure, compounds are predicted to bind in five regions: grooves A, B, C, D, and E. (C) Ten (10) binding sites considered, taking all 12 structures into account.

High-Throughput Docking. The docking of compound Subset A in 12 FXa conformations (i.e., the crystal structure and another 11 representative structures from 15 clusters obtained from $\mathrm{MD}$ ) represented a total of $\sim 2.9$ million docking calculations (Step II in Figure 2). After selecting the best scoring poses of docked ligands, as predicted by AutoDock Vina, and parsing these for the selected FXa-FVa binding sites, it was found that, in the crystal structure, most of the compounds (99.8\%) bind to sites outside of the FXa/FVa interface, mostly in the protein active site, and only $\sim 0.2 \%$ of compounds screened ( 532 compounds in the case of the crystal structure) bind to FXa within the FXa/FVa interface. As the MD snapshots sample larger conformational spaces, more compounds bind at this interface, compared to the number that bind to the crystal structure. Docking of Subset B compounds in the 12 structures represented another $\sim 0.45$ million docking calculations (Step VI in Figure 2). In the case of crystal structure, only 177 of these compounds (0.5\%) bind to FXa within the $\mathrm{FXa} / \mathrm{FVa}$ interface, and the higher hit rate compared to Subset A could be originating from the larger MWs of Subset $B$ compounds. The number of compounds from Subset A and Subset B binding within the FXa/FVa interface in the MD snapshots is provided in the Supporting Information. Figure 4A shows the comparison between the predicted binding modes of a compound from Subset A (SBC-160,014) and one from Subset B (SBC-160,045) binding in the FXa/FVa interface in the crystal structure (both compounds were subsequently identified as inhibitors). As one would expect, larger compounds are found to bind to larger regions on the FXa surface, and also often (although not in all cases) exhibit better binding (docking) scores than smaller compounds. The structures of both these compounds are provided in the Supporting Information. 
Binding Pockets at the FXa/FVa Interface. In the crystal structure, the compounds that bind in the FXa/FVa interface were found to bind essentially in five regions, represented by five grooves, shown in Figure 4B. Taking the docking results from all snapshots together, the interface was divided into 10 sub-binding sites, as shown in Figure $4 \mathrm{C}$ and Table 1, with sub-

Table 1. Ten Sub-binding Sites Identified at the FXa/FVa Interface and the Corresponding FXa Residues in These Sites That Are Proposed to Interact with FVa (as shown in Figure 1A)

\begin{tabular}{cl}
$\begin{array}{c}\text { sub-binding } \\
\text { site }\end{array}$ & \multicolumn{1}{c}{ residues } \\
1 & Arg-125, Asp-126, Trp-127 \\
2 & Val-231, Leu-235 \\
3 & Val-231, Ile-238 \\
4 & Arg-125, Asp-126, Val-231, Thr-232, Ala-233, Phe-234, Leu-235, \\
& Lys-236, Trp-237, Ile-238, Asp-239, Arg-240 \\
5 & Glu-129, Thr-232, Ala-233, Phe-234, Leu-235, Lys-236, Trp- \\
& 237, Ile-238 \\
6 & Ser-241, Met-242, Lys-243, Arg-93, Phe-94 \\
7 & Arg-93, Phe-94, Thr-95, Lys-96 \\
8 & Arg-93, Phe-94, Thr-95, Lys-96 \\
9 & Arg-125, Asp-126, Trp-127, Ala-128, Glu-129, Asp-164, Arg-165, \\
& Asn-166, Ser-167, Cys-168, Lys-169, Leu-170, Val-231, Thr- \\
& 232, Ala-233 \\
10 & Asp-164, Arg-165, Asn-166, Ser-167, Cys-168, Lys-169, Leu-170
\end{tabular}

binding sites $1,4,5,6$, and 9 corresponding to grooves A, B, C, $\mathrm{D}$, and $\mathrm{E}$ in the crystal structure, respectively. Other binding sites were not observed in the crystal structure but only in MD snapshots. The 10 binding subsites are thus defined by the localization of compounds observed in the docking studies, as opposed to the topological features of the protein surface as observed in the crystal structure. It was found that compounds with top snapshot count scores were concentrated in subbinding sites $9(52 \%), 7(27 \%)$, and $10(4 \%)$, respectively. Compounds with low binding-site scores of 1 and 2 mostly bind to sub-binding site 9 as well. This site is relatively large and present at the center of the binding interface.

The strongest binders, as predicted by Vina docking scores, were also concentrated in sub-binding sites 9 (43\%), 7 (38\%), and $10(22 \%)$. These compounds are weighted heavily from
Subset B ( 91\%) — compounds that arose from the pharmacophore-based search around the best-ranked compounds from Subset A-and represent an overall higher molecular weight than that of compounds in Subset A.

Experimental Validation. After employing further filters to the compounds in the two lists based on the compounds' availability, cost, chemical similarity, and presence of reactive functional groups, compounds were obtained for experimental validation, shown in Step IX in Figure 2. The majority of these selected compounds were predicted to bind in sites 9 and 7 , with some representation of the remaining binding sites. Of the first 97 compounds obtained for study, only 41 were fully soluble in the standard assay buffer. The soluble compounds were screened for their effect on PTase and FXa enzyme activity. Initial screening of these compounds at a concentration of $100 \mu \mathrm{M}$ was performed in a PTase activity assay measuring the inhibition of the rate of generation of active thrombin, using the thrombin specific chromogenic substrate, S-2238. PTase activity assays identified 19 of the 41 tested compounds as inhibitors, giving a success rate of $46 \%$. Ten (10) of the 19 inhibitors were predicted to bind well in one or more of the MD snapshots, but they did not bind to the crystal structure; these inhibitors would not have been identified if the docking calculations had been made using only the crystal structure. A chemical database search identified 22 structural analogues of SBC-160,042, (Steps $\mathrm{Xa}$ and $\mathrm{Xb}$ in Figure 2). Nine of these inhibited PTase activity. Five analogues of SBC-160,029 were also found, yielding 3 additional inhibitors. From the three sets of purchased compounds, a total of 31 inhibitors were identified: 19 were derived from the computational screen and 12 were based on structural homology without computational docking.

These compounds inhibit PTase activation of thrombin from prothrombin in the presence of FVa, but when assayed as direct FXa inhibitors using the FXa chromogenic peptide substrate, S2222 , the compounds were not inhibitory, indicating that they do not bind to the active site of the FXa molecule. Control experiments also showed that the compounds do not inhibit thrombin activity toward its chromogenic substrate, S-2238. The compounds thus appear to be specific inhibitors of the $\mathrm{FXa}-\mathrm{FVa}$ activation of prothrombin, and this inhibition is dependent upon the interaction between FXa and FVa.
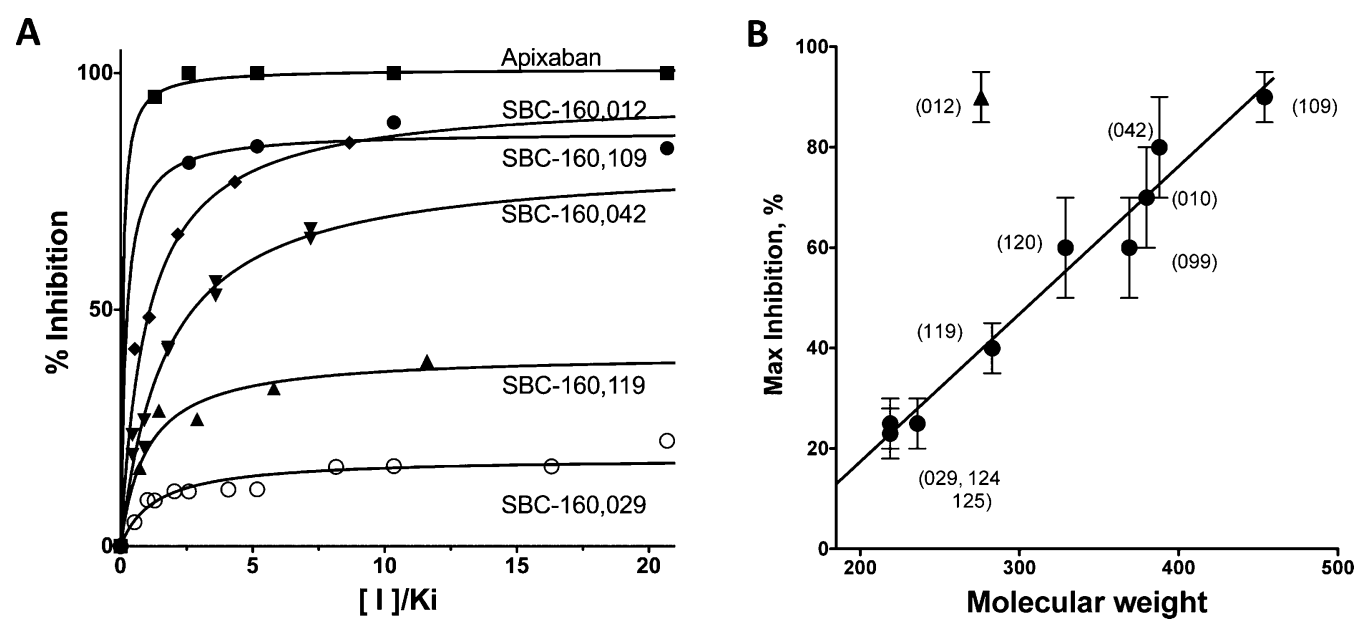

Figure 5. PTase inhibition. (A) Effect of the identified inhibitors on PTase activity, based on activity measurements, presented as \% inhibition vs [I]/ $K_{\mathrm{i}}$. (B) Plot of the maximum inhibition versus the molecular weights of identified PTase self-limiting inhibitors from Table 2. 
In order to determine the affinities of the compounds and their maximal inhibition at the saturating inhibitor, the compounds were assayed as a function of inhibitor concentration at fixed enzyme and substrate concentrations. Ten (10) of the 31 compounds exhibited a plateau with only partial or incomplete inhibition of PTase activity as the inhibitor concentration was increased. As shown in Figure 5A, the extent of inhibition reaches plateau levels of inhibition, in some cases well below the maximum level of $100 \%$. This plateau level of inhibition was maintained even though the inhibitor concentration was increased to 10 or 20 times the calculated $K_{\mathrm{i}}$ value. The remaining 21 compounds, although inhibitory, did not achieve a plateau inhibition at an inhibitor concentration of $100 \mu \mathrm{M}$. The binding affinities of these are not tight enough to accurately determine the maximal level of inhibition or the $K_{\mathrm{i}}$ value. Figure 5A, which shows the percentage inhibition as a function of $[I] / K_{i}$, contrasts the effects of five of the partial inhibitors on PTase activity with the effect of the anticoagulant apixaban, which is an active site competitive inhibitor of FXa. Whereas apixaban completely inhibits activity as the inhibitor concentration is increased, the characteristic feature of these novel inhibitors is that they achieve or approach plateau levels of inhibition below that observed with apixaban. Maximal inhibition, calculated from the inhibition curves, is $100 \%$ for apixaban, $25 \%$ for SBC-160,029, $40 \%$ for SBC-160,119, $85 \%$ for SBC-160,042, and $90 \%-95 \%$ for SBC-160,109/SBC-160,012.

SBC-160,029 was identified by the computational screen. It is a member of a substituted tetrazole series and binds to FXa in four snapshots, including the crystal structure. SBC-160,029 docked exclusively in site 9. SBC-160,029 inhibits PTase activity to a maximum of $\sim 25 \%$, with a $K_{\mathrm{i}}$ value of $15 \mu \mathrm{M}$ (Table 2). Two analogues-SBC-160,124 and SBC-160,125 (see Table 2)-exhibit similar maximal inhibitory activity, with $K_{\mathrm{i}}$ values of 5 and $20 \mu \mathrm{M}$, respectively. SBC-160,120, which is a larger analogue of SBC-160,029, achieved a plateau inhibition of $60 \%$ with $K_{\mathrm{i}}=30 \mu \mathrm{M}$.

Compounds SBC-160,012 and SBC-160,042 were also identified as site 9 binders, but both compounds also dock into site 7 in other MD snapshots. SBC-160,012 docks in site 9 in the crystal structure and two other snapshots, but docks to site 7 in snapshot 10 . SBC-160,042 does not dock in the crystal structure, but docks in site 9 in one snapshot and in site 7 in another. As listed in Table 2, the 10 partial inhibitors exhibit $K_{\mathrm{i}}$ values of 5-40 $\mu \mathrm{M}$, but the maximal inhibition for each compound varies from $23 \%$ (SBC-160,124) to $90 \%$ (SBC160,012 , SBC-160,109). Comparing the series of inhibitors in Table 2 indicated that maximal inhibition increased with increasing size of the inhibitor. Figure $5 \mathrm{~B}$ shows the change in maximum PTase inhibition with the molecular weights of the compounds. It can be seen that larger compounds inhibit PTase activity to a greater extent. SBC-160,109, which is a relatively tight binding analogue of SBC-160,042 with higher molecular weight, shows greater inhibition, contrasted with SBC-160,119, which is another SBC-160,042 analogue with lower molecular weight that exhibits lower maximal inhibition, compared to SBC-160,042. Interestingly, SBC-160,012, which has a relatively small molecular weight, is a clear outlier from this trend, suggesting a complex inhibition mechanism for this compound.

These hits comprise four families of inhibitors, as represented by SBC-160,010, SBC-160,029, SBC-160,012, and SBC160,042 , respectively. SBC-160,099, SBC-160,109 and SBC160,119 were selected from a panel of structural analogues of
Table 2. Ten Self-Limiting Inhibitors Identified by PTase Activity Assays $a, b$

\begin{tabular}{|c|c|c|c|c|c|c|}
\hline $\begin{array}{c}\text { SBC } \\
\text { ID }\end{array}$ & Structure & $\begin{array}{c}\text { Max \% } \\
\text { inhibition }\end{array}$ & $\begin{array}{l}\mathbf{K i}, \\
\mu \mathrm{M}\end{array}$ & $\begin{array}{c}\text { Binding } \\
\text { Snapshots }\end{array}$ & $\begin{array}{l}\text { Binding } \\
\text { Sites }\end{array}$ & $\begin{array}{r}\text { Binds } \\
\text { Xtal }\end{array}$ \\
\hline $\begin{array}{c}\text { SBC- } \\
160,010\end{array}$ & & $70 \pm 10$ & $20 \pm 6$ & 3 & $7 / 8 *$ & No \\
\hline $\begin{array}{c}\text { SBC- } \\
160,012\end{array}$ & & $90 \pm 5$ & $10 \pm 5$ & $1,2,4,10$ & $9,9,9,7$ & Yes \\
\hline $\begin{array}{c}\text { SBC- } \\
160,029\end{array}$ & & $25 \pm 5$ & $15 \pm 5$ & $1,2,5,9$ & $9,9,9,9$ & Yes \\
\hline $\begin{array}{c}\text { SBC- } \\
160042\end{array}$ & & $80 \pm 10$ & $20 \pm 10$ & 4,10 & 9,7 & No \\
\hline${ }^{\dagger} \mathrm{SBC}-$ & & $60 \pm 10$ & $40 \pm 10$ & 8 & 10 & No \\
\hline $\begin{array}{c}{ }^{\dagger} \mathrm{SBC}- \\
160,109\end{array}$ & & $90 \pm 5$ & $5 \pm 3$ & 3 & 7 & No \\
\hline $\begin{array}{c}{ }^{\dagger} \text { SBC- } \\
160,119\end{array}$ & & $40 \pm 5$ & $10 \pm 5$ & 5 & 9 & No \\
\hline $\begin{array}{c}{ }^{\ddagger} \mathrm{SBC}- \\
160,120\end{array}$ & & $60 \pm 10$ & $30 \pm 10$ & 2,5 & 9,10 & No \\
\hline $\begin{array}{l}{ }^{ \pm} \text {SBC- } \\
160,124\end{array}$ & & $23 \pm 5$ & $5 \pm 3$ & 2,5 & 9,9 & No \\
\hline $\begin{array}{l}{ }^{\ddagger} \text { SBC- } \\
160,125\end{array}$ & & $25 \pm 5$ & $20 \pm 10$ & 2,5 & 9,9 & No \\
\hline
\end{tabular}

${ }^{a}$ The compounds noted as structural analogues were later subjected to the virtual screen to identify their hypothetical binding sites. Max \% inhibition and $\mathrm{Ki}$ were obtained by fitting activity inhibition as a function of inhibitor concentration. Binding snapshot 1 represents the crystal structure. ${ }^{b}$ Symbols used within table: asterisk $(*)$ denotes binding between sites 7 and 8 ; dagger $(\dagger)$ indicates data selected as an analogue of SBC-160,042; and double dagger $(\ddagger)$ indicates data selected as an analogue of SBC-160,029.

SBC-160,042, whereas SBC-160,120, SBC-160,124, and SBC160,125 are analogues of SBC-160,029. The structures of these compounds are provided in Table 2.

In summary, from $\sim 3.4$ million docking calculations, the computational results yielded 535 compounds that could 
potentially bind to FXa within the FXa-FVa interaction region. Thirty one (31) inhibitors were identified, with 10 of them being represented by four families of inhibitors, exhibiting partial inhibition of PTase activity in which the extent of inhibition reaches a maximal plateau value as the enzyme complex is saturated with inhibitor, so that further increases in inhibitor concentration do not further inhibit PTase enzyme activity. We refer to this as "self-limiting inhibition". Four of these self-limiting inhibitors originated directly from the initial docking calculations; the remaining six were selected as structural analogues of SBC-160,029 and SBC-160,042.

Sub-binding Sites Analysis. Table 2 shows four families of inhibitors, represented by parent compounds SBC-160,010, SBC-160,012, SBC-160,042, and SBC-160,029. These compounds were identified as nonactive site inhibitors by PTase and FXa activity assays. Compounds SBC-160,010 and SBC160,029 bind in only sites $7 / 8$ (binding between sites 7 and 8 ) and 9, respectively, whereas SBC-160,012 and SBC-160,042 are able to potentially bind in either site 9 or site 7 in separate snapshot structures. Table 3 lists the difference in predicted

Table 3. Differences in Binding Free Energy for Selected Compounds in Binding Site 9 and Binding Site 7 in Snapshot 10

$\begin{array}{cc}\text { compound } & \Delta \Delta G(\Delta G \text { site } 7-\Delta G \text { site } 9)(\mathrm{kcal} / \mathrm{mol}) \\ \text { SBC-160,012 } & -3.72 \\ \text { SBC-160,042 } & +2.38\end{array}$

binding energies calculated using the MM/GBVI protocol in MOE for the two compounds binding in site 7 and in site 9 in snapshot 10 . The results indicate that compound SBC-160,012 is predicted to bind more strongly in binding site 7 than in binding site 9. Compound SBC-160,042, however, is predicted to bind more strongly in binding site 9 .

During clustering of the MD trajectory, some clusters showed relatively higher population of structures, compared to the others. Table 4 gives the cluster population from which

Table 4. Cluster Population for the Binding Snapshots of Compounds SBC-160,010, SBC-160,012, SBC-160,029, and SBC-160,042

$\begin{array}{cc}\text { snapshot number } & \text { cluster population (in \% of MD structures) } \\ 2 & 10.7 \\ 3 & 0.2 \\ 4 & 4 \\ 5 & 1.3 \\ 9 & 2.3 \\ 10 & 0.5\end{array}$

the representative snapshots (centroid structures) were selected. Among the snapshots to which these compounds bind, site 9 was identified in snapshots 2 and 4, and these show relatively higher cluster populations $(10.7 \%$ and $4 \%$, respectively), whereas binding site 7 was identified only in snapshot 10 that represents a cluster of structures that was found only $0.5 \%$ of the time in the MD trajectory. Snapshot 10 thus represents structures rarely sampled making binding in site 7 for compound SBC-160,012 a rare but possible binding pose, which is nonetheless quite significant, in terms of the strength of the interaction $(\Delta \Delta G=-3.72)$.
The major structural changes taking place during the MD trajectory are seen in loop L1 and helices $\mathrm{H} 2$ and $\mathrm{H} 3$, respectively (see the Supporting Information). On the other hand, helix $\mathrm{H1}$, which was present at the interface, remained fairly stable throughout the simulation. Site 9 was observed in the crystal structure, but its shape and structure change significantly during the MD simulation. Figure $6 \mathrm{~A}$ shows the

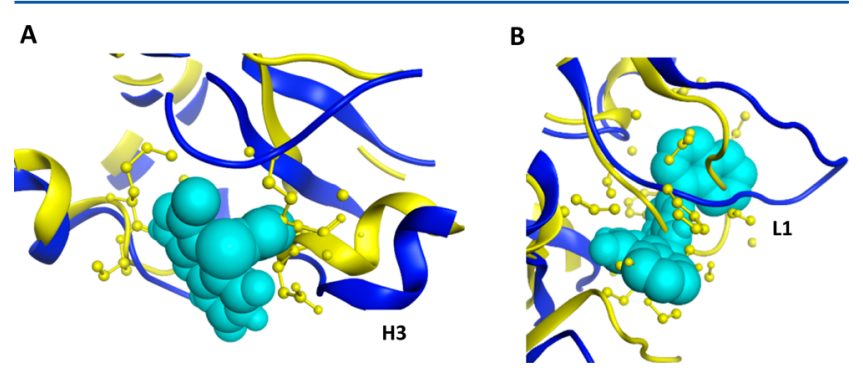

Figure 6. Structural variations with MD. Superposition of loop backbones in the $\mathrm{FXa}-\mathrm{FVa}$ interacting region. The FXa crystal structure is shown in yellow, whereas snapshots from the $\mathrm{MD}$ trajectory are shown in blue. (A) SBC-160,042 (cyan) binds to subbinding site 9 in snapshot 4, where the helix $3(\mathrm{H} 3)$ is pushed out, and not in the crystal structure. (B) SBC-160,010 (cyan) binds to subbinding site $7 / 8$ in snapshot 3 , where the loop 1 (L1) moves out, opening the binding site.

predicted binding mode of a compound identified initially as an inhibitor that binds to site 9 only in the later snapshots where the helix $\mathrm{H} 3$ is pushed out and not in the crystal structure. Subbinding site 7 was not seen in the crystal structure and became apparent in 3 of the 11 snapshots from the MD trajectory. Figure $6 \mathrm{~B}$ shows compound SBC-160,010, which is one of the compounds initially found as a nonactive site binder docked in snapshot 3 , and how it is not possible for it to bind to the crystal structure since loop L1 occludes the binding site in the crystal structure but moves out during $\mathrm{MD}$ trajectory, forming this site. SBC-160,010, inhibits PTase activity by $\sim 70 \%$.

\section{DISCUSSION}

Results from clustering the MD trajectory indicate that (i) the regions of FXa that interact with FVa sample a relatively large accessible conformational space, as seen in Figures 3A and 3C, and (ii) ligands are predicted to interact with FXa in a way that would not necessarily be observed from an analysis that focused solely on the starting crystal structure. These structural variations shown in the MD translated into a diverse set of compounds that potentially bind to the conformations of FXa.

The MD trajectory shows substantial structural changes taking place at the $\mathrm{FXa} / \mathrm{FVa}$ interface. The different conformations sampled by FXa may correspond to different structures of FXa that can possibly interact with and bind to $\mathrm{FVa}$, or the protein conformations that are in the process of equilibration in the FXa-FVa complex. It is also possible that PT interacts with some regions of loop L1 when binding to the active site. There are several well-conserved electrostatic residues present in the loop that may be involved in longrange interactions with $\mathrm{PT}$, and the flexibility of this loop may allow PT to "fit" in the FXa binding site.

The novel agnostic docking approach utilized in this study identified 10 possible binding pockets at the $\mathrm{FXa} / \mathrm{FVa}$ interface, shown in Figure 4C. Sub-binding site 9, a relatively large area at the center of the FVa binding interface, was found to be a major binding site for compounds. Most of the nonactive site 
inhibitors identified by PTase activity assays are predicted to bind to site 9, indicating the importance of this site for the interaction of FXa with FVa. Compounds that bind at this site interact with residues from all three helices of interest $(\mathrm{H} 1, \mathrm{H} 2$, and H3) and inhibit PTase activity. This site was observed in the crystal structure, but its shape and structure change significantly during the MD simulation. Figure 6A shows compound SBC-160,042 that was identified as a self-limiting inhibitor with a maximal inhibition of PTase activity at saturating concentrations of $\sim 80 \%$. SBC-160,042 is predicted to bind in site 9 , but the binding would not have been identified from the crystal structure searches alone.

The binding studies indicated that some compounds had the potential of binding in more than one site. SBC-160,012 and SBC-160,042 are two notable examples, and both compounds are capable of binding in sites 7 and 9 in different snapshot structures. Tables 3 and 4 indicate that, while site 9 (snapshots $2,4,5$, and 9) is sampled $\sim 3$ to $\sim 20$-fold more often than site 7 (snapshot 10), the predicted binding affinity of compound SBC-160,012 (predicted to bind in both sites 7 and 9) is stronger in site 7 by $3.72 \mathrm{kcal} / \mathrm{mol}$. Hence, this compound's predicted binding site is difficult to assess: site 9 is more often accessible but binding in site 7 is more stable. It is theoretically possible that the compound may bind at both sites and possibly simultaneously. Inversely, binding of compound SBC-160,042 is predicted to bind most likely in binding site 9, since the predicted binding free energy of this compound is stronger in site 9 than in site 7 , by $2.38 \mathrm{kcal} / \mathrm{mol}$.

From the crystal structure of the prothrombinase complex, from the venom of $P$. textilis, ${ }^{28}$ it can be observed that there are binding pockets present at the interface of FX and FV that could possibly be targeted by small molecules. Figures $7 \mathrm{~A}$ and $7 \mathrm{~B}$ show the superposition of human FXa (crystal structure) bound with a site 9 inhibitor (SBC-160,045) in its predicted binding mode, with the FX-FV complex from $P$. textilis. The similarity of the $P$. textilis structure and the human FXa structure suggests that the binding sites investigated here for human FXa are relevant to the modulation of FXa-FVa interaction. Site 9 binding compounds discovered from the agnostic computational approach bind directly at the interface of FXa and the A2/A3 domains of FV. The observation that these compounds inhibit PTase activity supports the hypothesis that they may interfere with the specific interactions between these proteins. Figures $7 \mathrm{C}$ and $7 \mathrm{D}$ show the superposition of the $P$. textilis FX-FV complex with human FXa (snapshot 10 from MD simulation) containing the inhibitor SBC-160,062, which inhibits activity by $20 \% \pm 7 \%$ and is predicted to bind in site 7. In this snapshot, the flexible loop L1 folds to form site 7, which is not seen in the crystal structure of human or $P$. textilis FXa. As seen in this MD snapshot, binding of ligands in site 7 can interfere with the interactions between human FXa and the small hydrophobic portion of the terminal region in FV. The structures of these compounds are given in the Supporting Information.

The initial objective of this study was to determine if an agnostic virtual screening approach could be used to identify potential binding sites on FXa for compounds that could inhibit PTase activity. This objective has been met as shown in Figure $5 \mathrm{~A}$ and Table 2. Larger compounds are found to bind to larger sites on the FXa surface, with docking scores that predict tighter binding compared to smaller compounds, which could prove advantageous in modulating the $\mathrm{FXa}-\mathrm{FVa}$ protein-
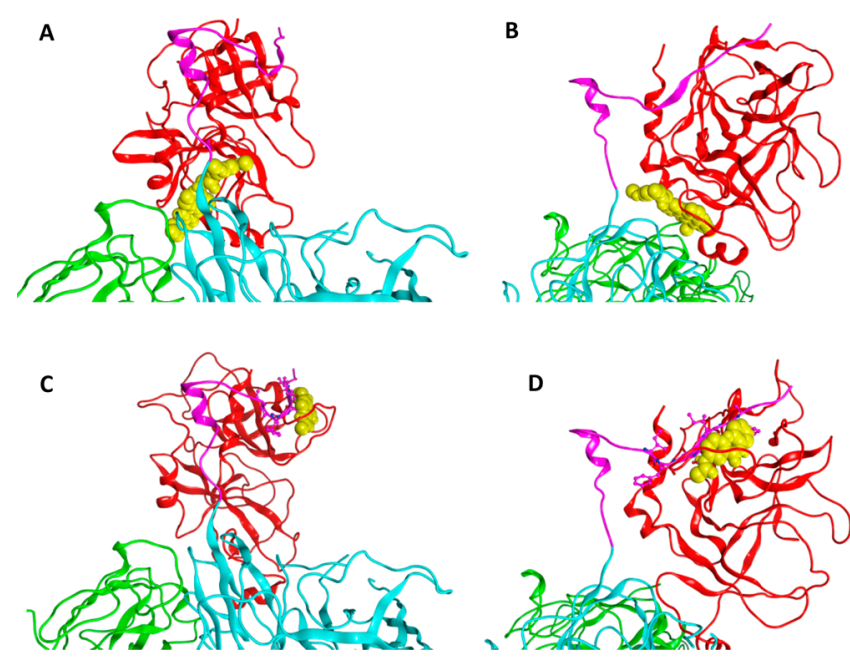

Figure 7. Site 9 and 7 in FX-FV complex. (A) Superposition of human FXa (crystal structure) bound with a site 9 inhibitor (SBC-160,045), with the FX-FV complex from $P$. textilis. Only superposed human FXa and $P$. textilis FV are shown, and the $P$. textilis FX structure is not shown for the sake of clarity. The human FXa structure is shown in red; the A2 domain from $P$. textilis FV is shown in cyan; the A3 domain from $P$. textilis FV is shown in green; and the flexible terminal region in FV is shown in purple. The site 9 inhibitor binds directly at the interface of $\mathrm{FXa}$ and $\mathrm{A} 2 / \mathrm{A} 3$ domains of $\mathrm{FV}$. (B) $90^{\circ}$ rotation of the structure shown in panel (A). (C) Superposition of human FXa (snapshot 10 from MD simulation) bound with site 7 inhibitor (SBC160,062), with the FX-FV complex (the hydrophobic portion of the terminal region is shown in stick conformation). (D) $90^{\circ}$ rotation of the structure shown in panel (C).

protein interaction. Figure 5B supports the hypothesis in that larger compounds are better inhibitors of PTase activity.

In future studies, lead optimization can be aided by the chemical structures and binding poses of positive hits predicted to bind at sites 7, 9, and 10. Library design can be based on compound modifications guided by pharmacophore features associated with active compounds while, at the same time, avoiding those features associated with inactive compounds (e.g., volume exclusion). It should also be possible to differentiate between binding in site 7 and site 9 from the experimental testing of compounds originating by crystallography, as well as by directed mutagenesis studies that would affect the predicted potential binding modes identified computationally. These compounds will be used to identify new hits and to develop structure-activity relationships (SARs) in order to identify more potent and selective lead compounds against PTase.

\section{CONCLUSION}

This study has used an ensemble docking approach to identify structural variations of FXa using molecular dynamics (MD) simulations, and it has targeted these conformations with compounds that specifically bind to the $\mathrm{FXa} / \mathrm{FVa}$ interface to modulate the PTase activity in a nonactive site-dependent manner. In contrast to competitive active site inhibitors, which completely inhibit enzyme activity at saturating inhibitor concentrations, these nonactive site PTase inhibitors exhibit a plateau in inhibition that is referred to as "self-limiting inhibition". PTase activity assays identified a total of 31 compounds as inhibitors. From these nonactive site inhibitors, 10 compounds achieved self-limiting inhibition of PTase, 8 of 
which would not have been identified using only the crystal structure of the protein.

This study exemplifies a successful MD simulation and highthroughput virtual screening approach to drug discovery targeting a protein-protein complex. Amaro et al. showed the importance of employing $\mathrm{MD}$ to account for protein flexibility in the discovery of new molecules for African sleeping sickness. ${ }^{51}$ Here, we apply a similar ensemble docking approach, but use a larger compound database, covering a larger chemical space, with $\sim 3.4$ million docking calculations completed in total. In future studies, the same approach can be extended to even larger compound databases, covering millions of compounds and targeting multiple protein targets, using massive high-throughput screenings on supercomputers such as TITAN. ${ }^{52,53}$ This can increase dramatically the number and diversity of compounds that can be identified while, at the same time, taking into account the inherent flexibility and the dynamic nature of macromolecules and their complexes under physiological conditions.

\section{ASSOCIATED CONTENT}

\section{S Supporting Information}

The Supporting Information is available free of charge on the ACS Publications website at DOI: 10.1021/acs.jcim.5b00596.

Number of compounds from Subset A and Subset B binding within the $\mathrm{FXa} / \mathrm{FVa}$ interface (Table $\mathrm{S} 1$ ); superimposed structures of human FXa and FX from $P$. textilis (Figure S1); validation of docking sampling/ accuracy vs docking speed (section S1); percentage similarity at different exhaustiveness values (Figure S2); superposition of crystal structure and MD snapshot (Figure S3); structural information for compounds shown in Figures 4 and 7 (Figure S4) (PDF)

\section{AUTHOR INFORMATION}

\section{Corresponding Authors}

*E-mail: michael@shifabiomedical.com (M. Blackburn).

*E-mail: jbaudry@utk.edu (J. Baudry).

\section{Author Contributions}

${ }^{\nabla}$ Contributed equally to this work.

\section{Author Contributions}

K.K. and N.M. performed the computational research and experiments; H.V.M. and M.N.B. acquired the compounds and performed the PTase assays; K.K., C.B.P., H.V.M., M.N.B., and J.B. designed the research; and K.K., N.M., C.B.P., H.V.M., M.N.B., and J.B. analyzed results, made the figures, and wrote the manuscript.

\section{Notes}

The authors declare the following competing financial interest(s): M.N.B., N.M., and H.V.M. are employees of Shifa Biomedical, which is a privately held, for-profit corporation.

\section{ACKNOWLEDGMENTS}

This work was supported by National Institutes of Health Grant No. HL114261 from the National Heart Lung and Blood Institute. The authors would also like to thank the Genome Science and Technology (GST) program at the University of Tennessee for their support.

\section{REFERENCES}

(1) Davie, E. W.; Ratnoff, O. D. Waterfall Sequence for Intrinsic Blood Clotting. Science 1964, 145, 1310-1312.

(2) Kane, W. H.; Davie, E. W. Blood Coagulation Factors V and VIII: Structural and Functional Similarities and Their Relationship to Hemorrhagic and Thrombotic Disorders. Blood 1988, 71 (3), 539555 .

(3) Mann, K. G.; Nesheim, M. E.; Church, W. R.; Haley, P.; Krishnaswamy, S. Surface-Dependent Reactions of the Vitamin KDependent Enzyme Complexes. Blood 1990, 76 (1), 1-16.

(4) Davie, E. W.; Fujikawa, K.; Kisiel, W. The Coagulation Cascade: Initiation, Maintenance, and Regulation. Biochemistry 1991, 30, 10363-10370.

(5) Bianchini, E. P.; Orcutt, S. J.; Panizzi, P.; Bock, P. E.; Krishnaswamy, S. Ratcheting of the Substrate from the Zymogen to Proteinase Conformations Directs the Sequential Cleavage of Prothrombin by Prothrombinase. Proc. Natl. Acad. Sci. U. S. A. 2005, 102, 10099-10104.

(6) Nesheim, M. E.; Taswell, J. B.; Mann, K. G. The Contribution of Bovine Factor V and Factor Va to the Activity of Prothrombinase. J. Biol. Chem. 1979, 254, 10952-10962.

(7) Rosing, J.; Tans, G.; Govers-Riemslag, J. W.; Zwaal, R. F.; Hemker, H. C. The Role of Phospholipids and Factor Va in the Prothrombinase Complex. J. Biol. Chem. 1980, 255, 274-283.

(8) Borensztajn, K.; Peppelenbosch, M. P.; Spek, C. A. Factor Xa: At the Crossroads between Coagulation and Signaling in Physiology and Disease. Trends Mol. Med. 2008, 14, 429-440.

(9) Berman, H. M.; Westbrook, J.; Feng, Z.; Gilliland, G.; Bhat, T. N.; Weissig, H.; Shindyalov, I. N.; Bourne, P. E. The Protein Data Bank. Nucleic Acids Res. 2000, 28, 235-242.

(10) Steen, M.; Dahlback, B. Thrombin-Mediated Proteolysis of Factor V Resulting in Gradual B-Domain Release and Exposure of the Factor Xa-Binding Site. J. Biol. Chem. 2002, 277, 38424-38430.

(11) Toso, R.; Camire, R. M. Removal of B-Domain Sequences from Factor V Rather Than Specific Proteolysis Underlies the Mechanism by Which Cofactor Function Is Realized. J. Biol. Chem. 2004, 279, 21643-21650.

(12) Heeb, M. J.; Kojima, Y.; Hackeng, T. M.; Griffin, J. H. Binding Sites for Blood Coagulation Factor Xa and Protein S Involving Residues 493-506 in Factor Va. Protein Sci. 1996, 5, 1883-1889.

(13) Kalafatis, M.; Beck, D. O. Identification of a Binding Site for Blood Coagulation Factor Xa on the Heavy Chain of Factor Va. Amino Acid Residues 323-331 of Factor V Represent an Interactive Site for Activated Factor X. Biochemistry 2002, 41, 12715-12728.

(14) Kalafatis, M.; Xue, J.; Lawler, C. M.; Mann, K. G. Contribution of the Heavy and Light Chains of Factor Va to the Interaction with Factor Xa. Biochemistry 1994, 33, 6538-6545.

(15) Kojima, Y.; Heeb, M. J.; Gale, A. J.; Hackeng, T. M.; Griffin, J. H. Binding Site for Blood Coagulation Factor Xa Involving Residues 311-325 in Factor Va. J. Biol. Chem. 1998, 273, 14900-14905.

(16) Steen, M.; Villoutreix, B. O.; Norstrom, E. A.; Yamazaki, T.; Dahlback, B. Defining the Factor Xa-Binding Site on Factor Va by Site-Directed Glycosylation. J. Biol. Chem. 2002, 277, 50022-50029.

(17) Kittur, F. S.; Manithody, C.; Rezaie, A. R. Role of the NTerminal Epidermal Growth Factor-Like Domain of Factor X/Xa. J. Biol. Chem. 2004, 279, 24189-24196.

(18) Rezaie, A. R. Identification of Basic Residues in the HeparinBinding Exosite of Factor Xa Critical for Heparin and Factor Va Binding. J. Biol. Chem. 2000, 275, 3320-3327.

(19) Rezaie, A. R.; Kittur, F. S. The Critical Role of the 185-189Loop in the Factor Xa Interaction with $\mathrm{Na}^{+}$and Factor $\mathrm{Va}$ in the Prothrombinase Complex. J. Biol. Chem. 2004, 279, 48262-48269.

(20) Rudolph, A. E.; Porche-Sorbet, R; Miletich, J. P. Substitution of Asparagine for Arginine 347 of Recombinant Factor Xa Markedly Reduces Factor Va Binding. Biochemistry 2000, 39, 2861-2867.

(21) Rudolph, A. E.; Porche-Sorbet, R.; Miletich, J. P. Definition of a Factor Va Binding Site in Factor Xa. J. Biol. Chem. 2001, 276, 51235128. 
(22) Thiec, F.; Cherel, G.; Christophe, O. D. Role of the Gla and First Epidermal Growth Factor-Like Domains of Factor $\mathrm{X}$ in the Prothrombinase and Tissue Factor-Factor Viia Complexes. J. Biol. Chem. 2003, 278, 10393-10399.

(23) Yegneswaran, S.; Mesters, R. M.; Griffin, J. H. Identification of Distinct Sequences in Human Blood Coagulation Factor $\mathrm{Xa}$ and Prothrombin Essential for Substrate and Cofactor Recognition in the Prothrombinase Complex. J. Biol. Chem. 2003, 278, 33312-33318.

(24) Kamata, K.; Kawamoto, H.; Honma, T.; Iwama, T.; Kim, S. H. Structural Basis for Chemical Inhibition of Human Blood Coagulation Factor Xa. Proc. Natl. Acad. Sci. U. S. A. 1998, 95, 6630-6635.

(25) Adams, T. E.; Hockin, M. F.; Mann, K. G.; Everse, S. J. The Crystal Structure of Activated Protein C-Inactivated Bovine Factor Va: Implications for Cofactor Function. Proc. Natl. Acad. Sci. U. S. A. 2004, 101, 8918-8923.

(26) Autin, L.; Steen, M.; Dahlback, B.; Villoutreix, B. O. Proposed Structural Models of the Prothrombinase (Fxa-Fva) Complex. Proteins: Struct., Funct., Genet. 2006, 63, 440-450.

(27) Wallnoefer, H. G.; Handschuh, S.; Liedl, K. R.; Fox, T. Stabilizing of a Globular Protein by a Highly Complex Water Network: A Molecular Dynamics Simulation Study on Factor Xa. J. Phys. Chem. B 2010, 114, 7405-7412.

(28) Lechtenberg, B. C.; Murray-Rust, T. A.; Johnson, D. J.; Adams, T. E.; Krishnaswamy, S.; Camire, R. M.; Huntington, J. A. Crystal Structure of the Prothrombinase Complex from the Venom of Pseudonaja Textilis. Blood 2013, 122, 2777-2783.

(29) Phillips, J. C.; Braun, R.; Wang, W.; Gumbart, J.; Tajkhorshid, E.; Villa, E.; Chipot, C.; Skeel, R. D.; Kale, L.; Schulten, K. Scalable Molecular Dynamics with Namd. J. Comput. Chem. 2005, 26, 17811802.

(30) Griffon, N.; Di Stasio, E. Thermodynamics of $\mathrm{Na}^{+}$Binding to Coagulation Serine Proteases. Biophys. Chem. 2001, 90, 89-96.

(31) Underwood, M. C.; Zhong, D.; Mathur, A.; Heyduk, T.; Bajaj, S. P. Thermodynamic Linkage between the $\mathrm{S} 1$ Site, the $\mathrm{Na}^{+}$Site, and the $\mathrm{Ca}^{2+}$ Site in the Protease Domain of Human Coagulation Factor Xa. Studies on Catalytic Efficiency and Inhibitor Binding. J. Biol. Chem. 2000, 275, 36876-36884.

(32) Molecular Operating Environment (MOE); Chemical Computing Group: Montreal, Quebec, Canada, 2013.

(33) Labute, P. The Generalized Born/Volume Integral Implicit Solvent Model: Estimation of the Free Energy of Hydration Using London Dispersion Instead of Atomic Surface Area. J. Comput. Chem. 2008, 29, 1693-1698.

(34) MacKerell, A. D.; Bashford, D.; Bellott, M.; Dunbrack, R. L.; Evanseck, J. D.; Field, M. J.; Fischer, S.; Gao, J.; Guo, H.; Ha, S.; Joseph-McCarthy, D.; Kuchnir, L.; Kuczera, K.; Lau, F. T.; Mattos, C.; Michnick, S.; Ngo, T.; Nguyen, D. T.; Prodhom, B.; Reiher, W. E.; Roux, B.; Schlenkrich, M.; Smith, J. C.; Stote, R.; Straub, J.; Watanabe, M.; Wiorkiewicz-Kuczera, J.; Yin, D.; Karplus, M. All-Atom Empirical Potential for Molecular Modeling and Dynamics Studies of Proteins. J. Phys. Chem. B 1998, 102, 3586-3616.

(35) Bakan, A.; Meireles, L. M.; Bahar, I. Prody: Protein Dynamics Inferred from Theory and Experiments. Bioinformatics 2011, 27, $1575-1577$

(36) Shortle, D.; Simons, K. T.; Baker, D. Clustering of Low-Energy Conformations near the Native Structures of Small Proteins. Proc. Natl. Acad. Sci. U. S. A. 1998, 95, 11158-11162.

(37) Trott, O.; Olson, A. J. Autodock Vina: Improving the Speed and Accuracy of Docking with a New Scoring Function, Efficient Optimization, and Multithreading. J. Comput. Chem. 2010, 31, 455461.

(38) Irwin, J. J.; Sterling, T.; Mysinger, M. M.; Bolstad, E. S.; Coleman, R. G. ZINC: A Free Tool to Discover Chemistry for Biology. J. Chem. Inf. Model. 2012, 52, 1757-1768.

(39) Maggiora, G.; Vogt, M.; Stumpfe, D.; Bajorath, J. Molecular Similarity in Medicinal Chemistry. J. Med. Chem. 2014, 57, 31863204.
(40) Voigt, J. H.; Bienfait, B.; Wang, S.; Nicklaus, M. C. Comparison of the Nci Open Database with Seven Large Chemical Structural Databases. J. Chem. Inf. Model. 2001, 41, 702-712.

(41) O’Boyle, N. M.; Banck, M.; James, C. A.; Morley, C.; Vandermeersch, T.; Hutchison, G. R. Open Babel: An Open Chemical Toolbox. J. Cheminf. 2011, 3, 33.

(42) Halgren, T. A. Merck Molecular Force Field. I. Basis, Form, Scope, Parameterization, and Performance of Mmff94. J. Comput. Chem. 1996, 17, 490-519.

(43) Morris, G. M.; Huey, R.; Lindstrom, W.; Sanner, M. F.; Belew, R. K.; Goodsell, D. S.; Olson, A. J. Autodock4 and Autodocktools4: Automated Docking with Selective Receptor Flexibility. J. Comput. Chem. 2009, 30, 2785-2791.

(44) Koes, D. R; Camacho, C. J. ZincPharmer: Pharmacophore Search of the ZINC Database. Nucleic Acids Res. 2012, 40, W409W414.

(45) Brown, R. D.; Martin, Y. C. Use of Structure-Activity Data to Compare Structure-Based Clustering Methods and Descriptors for Use in Compound Selection. J. Chem. Inf. Model. 1996, 36, 572-584.

(46) Hemker, H. C.; Hemker, P. W. The Kinetics of Enzyme Cascade Systems. General Kinetics of Enzyme Cascades. Proc. R. Soc. London, Ser. B 1969, 173, 411-420.

(47) Corbeil, C. R.; Williams, C. I.; Labute, P. Variability in Docking Success Rates Due to Dataset Preparation. J. Comput.-Aided Mol. Des. 2012, 26, 775-786.

(48) Daura, X.; Haaksma, E.; van Gunsteren, W. F. Factor Xa: Simulation Studies with an Eye to Inhibitor Design. J. Comput.-Aided Mol. Des. 2000, 14, 507-529.

(49) Singh, N.; Briggs, J. M. Molecular Dynamics Simulations of Factor Xa: Insight into Conformational Transition of Its Binding Subsites. Biopolymers 2008, 89, 1104-1113.

(50) Venkateswarlu, D.; Perera, L.; Darden, T.; Pedersen, L. G. Structure and Dynamics of Zymogen Human Blood Coagulation Factor X. Biophys. J. 2002, 82, 1190-1206.

(51) Amaro, R. E.; Schnaufer, A.; Interthal, H.; Hol, W.; Stuart, K. D.; McCammon, J. A. Discovery of Drug-Like Inhibitors of an Essential Rna-Editing Ligase in Trypanosoma Brucei. Proc. Natl. Acad. Sci. U. S. A. 2008, 105, 17278-17283.

(52) Ellingson, S. R.; Dakshanamurthy, S.; Brown, M.; Smith, J. C.; Baudry, J. Accelerating Virtual High-Throughput Ligand Docking: Current Technology and Case Study on a Petascale Supercomputer. Concurr. Comput. 2014, 26, 1268-1277.

(53) Ellingson, S.; Smith, J. C.; Baudry, J. Polypharmacology and Supercomputer-Based Docking: Opportunities and Challenges. Mol. Simul. 2014, 40, 848-854. 